\title{
Controlling bleeding during uniportal thoracoscopic major pulmonary resection
}

\author{
Hitoshi Igai, Mitsuhiro Kamiyoshihara, Natsumi Matsuura, Fumi Ohsawa, Kazuki Numajiri \\ Department of General Thoracic Surgery, Japanese Red Cross Maebashi Hospital, Maebashi, Gunma 371-0811, Japan.
}

Correspondence to: Dr. Hitoshi Igai, Department of General Thoracic Surgery, Japanese Red Cross Maebashi Hospital, 389-1 Asakura-cho, Maebashi, Gunma 371-0811, Japan. E-mail: hitoshiigai@gmail.com

How to cite this article: Igai H, Kamiyoshihara M, Matsuura N, Ohsawa F, Numajiri K. Controlling bleeding during uniportal thoracoscopic major pulmonary resection. Mini-invasive Surg 2022;6:5. https://dx.doi.org/10.20517/2574-1225.2021.110

Received: 27 Sep 2021 First Decision: 2 Dec 2021 Revised: 7 Dec 2021 Accepted: 22 Dec 2021 Published: 17 Jan 2022

Academic Editor: Giulio Belli Copy Editor: Yue-Yue Zhang Production Editor: Yue-Yue Zhang

\begin{abstract}
Aim: In uniportal thoracoscopic major pulmonary resection, it is important to appropriately manage significant vessel injury, to ensure patient safety and minimize conversion to thoracotomy. We analyzed cases of significant vessel injury and investigated efficacy of an algorithm to manage bleeding during thoracoscopic uniportal major pulmonary resection.
\end{abstract}

Methods: A total of 169 patients underwent "uniportal thoracoscopic major pulmonary resection" (lobectomy or segmentectomy) at our department between February 2019 and April 2021. These patients were classified into groups with (group $A, n=8$ ) and without (group $B, n=161$ ) intraoperative massive bleeding. Patient characteristics and perioperative results were compared between the two groups. Patients with significant vessel injury and conversion to thoracotomy were analyzed in detail.

Results: Group B had significantly less blood loss (A: $197 \pm 173 \mathrm{~g} ; \mathrm{B}: 42 \pm 74 \mathrm{~g}, \mathrm{P}<0.0001$ ) and shorter-duration postoperative drainage ( $\mathrm{A}: 2.6 \pm 1.8$ days; $\mathrm{B}: 1.6 \pm 1.3$ days, $P=0.036)$. There were no group differences in any other factors. The most frequently injured vessel in group A was the pulmonary artery (75\%). Emergent conversion was required in four cases (cases $7,76,128$, and 133; $2.4 \%$ ) due to intraoperative bleeding. No patient developed catastrophic bleeding or required an intraoperative transfusion.

Conclusion: We managed significant vessel injury appropriately during uniportal thoracoscopic major pulmonary resection using the troubleshooting algorithm. The algorithm for the uniportal approach was considered effective and easy to apply even by less-experienced surgeons. 
Keywords: Troubleshooting, intraoperative bleeding, uniportal approach

\section{INTRODUCTION}

The first wedge resection through a single skin incision was reported in 1998 (not uniportal), and the first video-assisted thoracic surgery through a single port lung biopsy was reported in $2000^{[1,2]}$. Major pulmonary resection via a uniportal approach was introduced by Gonzalez et al. ${ }^{[3]}$ in 2011 . Subsequently, the use of this type of minimally invasive surgery has gradually increased worldwide. Our team started to use "uniportal thoracoscopic major pulmonary resection" in February 2019. The uniportal thoracoscopic approach is considered minimally invasive and has several advantages, including low postoperative pain, few morbidities, short-duration postoperative chest drainage, short hospitalization periods, rapid postoperative recovery, and relatively low medical costs ${ }^{[4-7]}$. Magouliotis et al. ${ }^{[8]}$ demonstrated more favorable short-term oncological results of a uniportal compared to multiportal approach for thoracic malignancies in their review article, although the long-term outcomes were unclear.

It is important to appropriately manage significant vessel injury causing intraoperative massive bleeding, to ensure patient safety and minimize conversion to thoracotomy. In previous reports, our team has advocated a troubleshooting algorithm to manage bleeding during multiportal thoracoscopic major pulmonary resection ${ }^{[9]}$. In the algorithm we often directly applied thrombostatic sealant to the vessel injury to achieve hemostasis. However, we expected this procedure was difficult during the uniportal approach for major pulmonary resection because the surgical instruments and thoracoscope were introduced via a single small skin incision. Therefore, we applied a modified algorithm suitable for a uniportal approach, which was necessary to ensure the safety of the uniportal approach for major pulmonary resection. Here, we analyzed cases with significant vessel injury and investigated efficacy of the modified algorithm to manage it during thoracoscopic uniportal major pulmonary resection.

\section{METHODS}

This study was conducted according to the Declaration of Helsinki (as revised in 2013). This study was approved by the Institutional Ethics Board of Japanese Red Cross Maebashi Hospital (Approval No.: 202127, Date: July 26, 2021) and patient consent for this retrospective analysis was waived.

Totally thoracoscopic major pulmonary resection was introduced in our institution in April 2012. The procedure was performed via three or four ports until February 2019, when the surgeon-author switched to uniportal thoracoscopic major pulmonary resection. Initially (first 30 cases), to ensure the safety of the operation, the uniportal approach was limited to $\mathrm{CT} 1 \mathrm{No}$ cases, pulmonary metastasis, or benign disease, a strategy that also allowed the surgical team to become familiar with this less invasive procedure. In all other major pulmonary resections, the use of a multiportal approach continued, until December 2019, when all patients were treated using the uniportal approach. A total of 250 patients underwent thoracoscopic major pulmonary resection (lobectomy or segmentectomy) between February 2019 and April 2021. Among them, 169 patients (67.6\%) underwent uniportal resection. The patients were classified into groups with (group A, $n=8$ ) and without (group B, $n=161$ ) significant vessel injury. The patient enrollment process is described in Figure 1. Significant vessel injury was defined as the injury of intrathoracic major vessels including pulmonary artery, pulmonary vein, azygos vein, superior vena cava, aorta, subclavian artery, or subclavian vein, which had the possibility of causing intraoperative life-threatening bleeding. Surgery via the uniportal approach was done by HI or NM, both of whom have performed more than 500 thoracoscopic major pulmonary resections via a multiportal approach. 


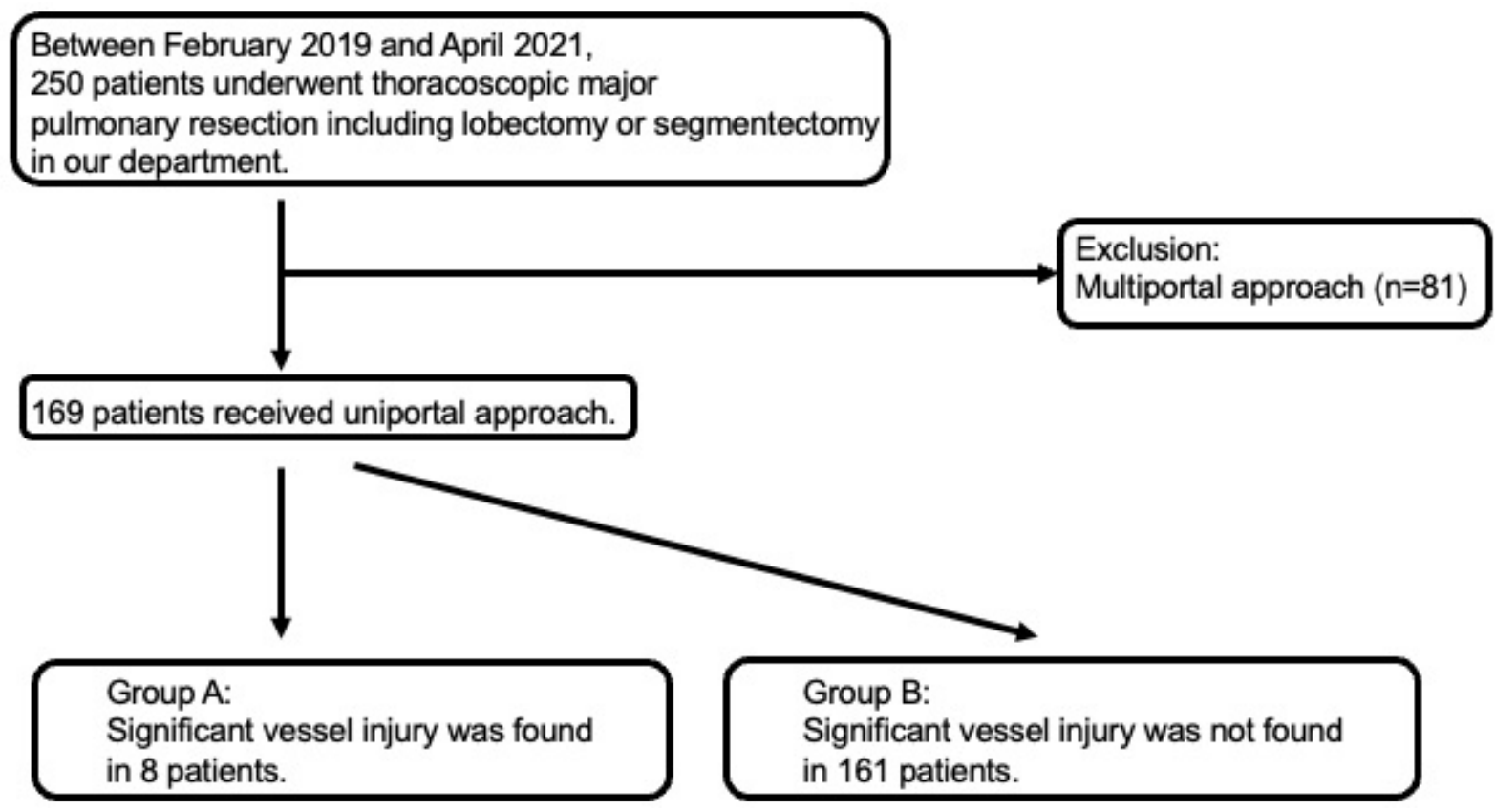

Figure 1. Patient enrollment.

The patient characteristics and perioperative results, including age, sex, treated lobe, disease, surgical procedure, operative time, blood loss, duration of postoperative drainage, postoperative hospitalization, morbidity (Clavien-Dindo grade $\geq$ III), readmission within 30 days after the operation, and 30-day mortality were compared between the two groups. Data on significant vessel injury (including from the injured vessel), the devices responsible for injury, the hemostatic procedure, the final procedure for the injured area, and conversion to thoracotomy were analyzed in detail. Ultimately, 10 of the 169 patients required conversion to thoracotomy. We reviewed these patients in detail.

\section{Surgical procedures}

Uniportal thoracoscopic major pulmonary resection was performed under general anesthesia using onelung ventilation with the patient in the lateral decubitus position. We placed a monitor above the head of the patient, and all procedures were performed under the monitor. A $3.5-4-\mathrm{cm}$ incision was made in the fourth or fifth intercostal space from the anterior axillary line. The access incision was covered with a size XXS wound retractor (Alexis Wound Retractor; Applied Medical, Rancho Santa Margarita, CA, USA). We inserted a 5- or 10- $\mathrm{mm}$ thoracoscope with a $30^{\circ}$ angle, and other surgical instruments, via this single incision in all patients. The large vessels and bronchi were divided with a stapler. Small-caliber vessels were divided with an energy device after proximal ligation. Specimens were placed in an endovascular bag and retrieved through the incision after completing the pulmonary resection. The incision was lengthened depending on the size of the specimen. We did not use a rib-spreader in any case. While systemic lymphadenectomy was performed in patients with primary lung cancer undergoing lobectomy, the hilar lymph nodes were sampled in patients with primary lung cancer undergoing segmentectomy. No lymphadenectomy was performed in patients with metastatic lung cancer or benign disease. When an air leak was found by the sealing test at the end of surgery, we applied polyglycolic acid felt (Neoveil, sheet type; Igaki Medical Planning Co., Ltd., Kyoto, Japan) to the air-leak point using fibrin glue (Beriplast P; CSL Behring, King of Prussia, PA, USA) with or without suturing with 3-0 absorbable monofilament. Finally, a 19-Fr chest drain (Blake Drain; Ethicon, Paramus, NJ, USA) or 24-Fr double-lumen chest tube was positioned in the thorax. 


\section{Significant vessel injury happens.}

Injured point is initially compressed with adjacent lung or a cotton stick. (Adjacent lung is better than a cotton stick.)

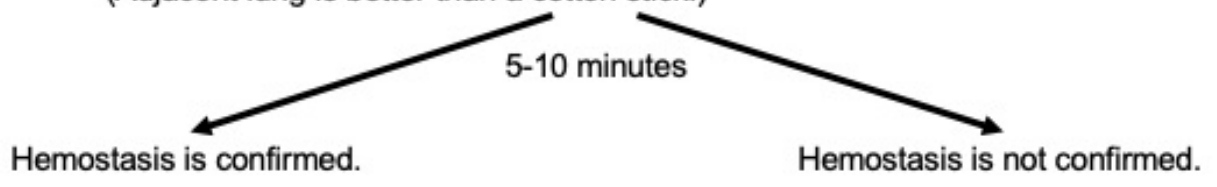

1. Thrombostatic sealant is attached to the injured point for reinforcement. (when the injured vessel should be preserved)

2. Stapling was performed (when the injured vessel should be divided)
1. Conversion to thoracotomy for proximal clamp

2. Conversion to multiport for attachment of thrombostatic sealant

3. Grasping, clipping or stapling via uniport or after the conversions (when the injured vessel is pulmonary vein, azygos vein, or subclavian vein.)

\footnotetext{
Notifications:

1: When total blood loss reached $\geqq 600 \mathrm{~g}$, do not hesitate to convert.

2: The injury of aortic arch or subclavian artery might be better to be consulted with cardiovascular surgeon.

3: The procedure of direct suturing thoracoscopically is not included in the algorithm.
}

Figure 2. Algorithm used in our department to manage bleeding during uniportal thoracoscopic major pulmonary resection.

Hemostatic procedures based on the algorithm

The algorithm to manage significant vessel injury during thoracoscopic uniportal major pulmonary resection was shown in Figure 2. The injured point is initially compressed with adjacent lung parenchyma or a cotton stick for 5-10 min. Although it depends on the situation, the adjacent lung parenchyma is generally better than a cotton stick because the latter can tear the injured area if it is not gently compressed. When hemostasis is confirmed by compression, the injured area is subsequently treated by applying thrombostatic sealant for reinforcement (when the injured vessel must be preserved) or by stapling (when the injured vessel must be divided). If hemostasis is not achieved with compression, thoracotomy or the multiportal approach is used instead of the uniportal approach. Conversion to thoracotomy was mainly performed for proximal clamp of the injured vessel. Conversion to multiport was mainly performed for attachment of thrombostatic sealant. The injured area could be directly grasped when the injured vessel was the pulmonary, subclavian, or azygos vein, although this was optional. The surgeon should choose the best option for the situation. When the injured vessel is the aortic arch or subclavian artery, a cardiovascular surgeon should be consulted because a pseudoaneurysm could develop postoperatively ${ }^{[10]}$. We did not include direct suturing of the injured vessel (via the uniportal or multiportal approach) in our algorithm, because it is technically difficult. Although a highly experienced thoracoscopic surgeon can likely suture appropriately even with the thoracoscopic approach, we attempted to construct an algorithm useful even for those inexperienced with uniportal thoracoscopic major pulmonary resection. Finally, we strongly insisted that we do not hesitate to convert when total blood loss reached $\geq 600 \mathrm{~g}$.

\section{Conversion to thoracotomy}

In several previous studies, conversion to thoracotomy was classified into two types: emergent and nonemergent ${ }^{[1-13]}$. Emergent conversion is done due to intraoperative massive bleeding or airway injury, which must be treated immediately by the surgical team. "Emergent conversion" should also be performed 
to avoid the injury becoming life-threatening. "Nonemergent conversion" is done in cases with pleural adhesions, calcified or inflammatory lymph node invasion to the pulmonary artery, or difficult-to-perform single lung ventilation (after careful intraoperative assessment). In this study, we classified the conversions into these two types.

\section{Statistical analysis}

We compared the patient characteristics and perioperative results between the two groups using the independent $t$-test or Fisher's exact test. A $P$-value $<0.05$ was considered significant. All analyses were performed using EZR (Saitama Medical Centre, Jichi Medical University, Saitama, Japan).

\section{RESULTS}

The patient characteristics and perioperative results of the two groups are listed in Table 1.

We found no significant differences in patient characteristics between the groups. Although group B had significantly less blood loss (A: $197 \pm 173 \mathrm{~g}$; B: $42 \pm 74 \mathrm{~g}, P<0.0001)$ and shorter-duration postoperative drainage (A: $2.6 \pm 1.8$ days vs. $1.6 \pm 1.3$ days, $P=0.036$ ), no significant differences were observed for the other perioperative parameters, including operative time (A: $149 \pm 41 \mathrm{~min}$; B: $145 \pm 46 \mathrm{~min}, P=0.85$ ), postoperative hospitalization time (A: $6 \pm 3.7$ days; B: $5.1 \pm 15.7$ days, $P=0.87$ ), Clavien-Dindo grade $\geq$ III (A: $11.1 \%$; $\mathrm{B}: 12.5 \%, P=1.00$ ), readmission within 30 days after the operation (A: $11.1 \%$; $\mathrm{B}: 3.7 \%, P=0.30$ ), or 30-day mortality (A: $0 \%$; $: 0.6 \%, P=1)$. No intraoperative transfusions were performed in any case.

Details of the cases with significant vessel injury are described in Table 2. The injured vessel was a branch of the pulmonary artery in six cases (75\%), a branch of the pulmonary vein in one case (12.5\%), and the azygos vein in one case (12.5\%). The device responsible for the vessel injury was a stapler in three cases (37.5\%), scissors in two cases (25\%), an "energy device" in two cases $(25 \%)$, and forceps in one case (12.5\%). Unfortunately, the surgical instruments were not used appropriately in any case, which resulted in the vessel injuries. The hemostatic procedure involved compression with the adjacent lung or a cotton stick in five cases (62.5\%; after conversion to thoracotomy in two cases of those five cases), proximal clamp after conversion to thoracotomy in two cases $(25 \%)$, and grasping by forceps in one case $(12.5 \%)$. The injured area was divided with a stapler in six cases $(75 \%)$ to remove the target lobe, while thrombostatic sealant was used to reinforce the injured area in two cases $(25 \%)$ to preserve the vessels. Four cases (50\%) required conversion to thoracotomy. The injured vessel was the pulmonary artery branch in the four cases, which was comprised of A2 (right), A6 (right), A3 (left), and A4+5 (left).

Figure 3 shows the cases requiring conversion to thoracotomy $(n=10)$. Nonemergent conversion was performed in six cases (due to severe pleural adhesions in one case, difficulty locating the tumor in one case, and difficulty encircling the pulmonary artery because of inflammatory lymph node invasion in four cases), and emergent conversion in four cases (all due to intraoperative bleeding; cases 7, 76, 128, and 133).

We have not encountered catastrophic intraoperative bleeding (i.e., > $1000 \mathrm{~g}$ of blood loss) since the uniportal approach was introduced for thoracoscopic major pulmonary resection. A patient in group B (without significant vessel injury) revealed $600 \mathrm{~g}$ blood loss, which was the maximum in this study.

Supplementary Video 1 demonstrated the procedural steps to achieve the hemostasis after the middle lobe of the pulmonary artery just behind the bronchus was injured by tip of the stapler during uniportal thoracoscopic middle lobectomy. We initially compressed the injured area using the adjacent middle lobe. 
Table 1. Patient characteristics and perioperative results

\begin{tabular}{|c|c|c|c|}
\hline Variables & $\begin{array}{l}\text { Group A } \\
(n=8, \%)\end{array}$ & $\begin{array}{l}\text { Group B } \\
(n=161, \%)\end{array}$ & P-value \\
\hline Age (year) & $72 \pm 15$ & $71 \pm 10$ & 0.94 \\
\hline Sex & & & 0.3 \\
\hline Female $(n)$ & $5(62.5)$ & $69(42.9)$ & \\
\hline Male $(n)$ & $3(37.5)$ & $92(57.1)$ & \\
\hline Treated lobe & & & 0.65 \\
\hline $\operatorname{LUL}(n)$ & $2(25)$ & $30(18.6)$ & \\
\hline $\operatorname{LLL}(n)$ & $1(12.5)$ & $21(13)$ & \\
\hline LUL and LLL $(n)$ & $0(0)$ & $2(1.2)$ & \\
\hline $\operatorname{RUL}(n)$ & $1(12.5)$ & $51(31.7)$ & \\
\hline $\operatorname{RML}(n)$ & $1(12.5)$ & $10(6.2)$ & \\
\hline $\operatorname{RLL}(n)$ & $3(37.5)$ & $47(29.2)$ & \\
\hline Disease & & & 0.32 \\
\hline Primary lung cancer $(n)$ & $6(75)$ & 139 (86.3) & \\
\hline Pulmonary metastasis $(n)$ & $1(12.5)$ & $7(4.3)$ & \\
\hline Benign disease $(n)$ & $1(12.5)$ & $15(9.3)$ & \\
\hline Surgical procedure & & & 0.52 \\
\hline Lobectomy (n) & $4(50)$ & $102(63.4)$ & \\
\hline Segmentectomy $(n)$ & $4(50)$ & $57(35.4)$ & \\
\hline Lobectomy and segmentectomy $(n)$ & $0(0)$ & $2(1.2)$ & \\
\hline Operative time ( $\mathrm{min}$ ) & $149 \pm 41$ & $145 \pm 46$ & 0.85 \\
\hline Blood loss (g) & $197 \pm 173$ & $42 \pm 75$ & $<0.0001$ \\
\hline Intraoperative transfusion $(n)$ & $0(0)$ & $0(0)$ & - \\
\hline Duration of postoperative drainage (days) & $2.6 \pm 1.8$ & $1.6 \pm 1.3$ & 0.037 \\
\hline Postoperative hospitalization (days) & $6 \pm 3.7$ & $5.1 \pm 15.7$ & 0.87 \\
\hline Morbidity (Clavien-Dindo grade $\geq \mathrm{III}$ ) (n) & $1(11.1)$ & $18(12.5)$ & 1 \\
\hline Readmission within 30 days after the operation & $1(11.1)$ & $6(3.7)$ & 0.3 \\
\hline 30-day mortality $(n)$ & $0(0)$ & $1(0.6)$ & 1 \\
\hline
\end{tabular}

LUL: Left upper lobe; LLL: left lower lobe; RUL: right upper lobe; RML: right middle lobe; RLL: right lower lobe.

Table 2. Details of patients with intraoperative massive bleeding

\begin{tabular}{|c|c|c|c|c|c|}
\hline $\begin{array}{l}\text { Case } \\
\text { No. }\end{array}$ & $\begin{array}{l}\text { Injured } \\
\text { vessels }\end{array}$ & $\begin{array}{l}\text { Devices responsible for } \\
\text { vessel injury }\end{array}$ & Hemostatic procedure & $\begin{array}{l}\text { Final procedure for the } \\
\text { injured area }\end{array}$ & Conversion \\
\hline 1 & PA branches & Stapler & Proximal clamp after conversion & Stapling & $\begin{array}{l}\text { To } \\
\text { thoracotomy }\end{array}$ \\
\hline 2 & PA branches & Scissors & Compression with a cotton stick & Stapling & Not performed \\
\hline 3 & PV branches & Stapler & Compression with adjacent lung & $\begin{array}{l}\text { Reinforced with thrombostatic } \\
\text { sealant }\end{array}$ & Not performed \\
\hline 4 & PA branches & Stapler & $\begin{array}{l}\text { Compression with adjacent lung } \\
\text { after conversion }\end{array}$ & $\begin{array}{l}\text { Reinforced with thrombostatic } \\
\text { Sealant }\end{array}$ & $\begin{array}{l}\text { To } \\
\text { thoracotomy }\end{array}$ \\
\hline 5 & PA branches & Forceps & Compression with adjacent lung & Stapling & Not performed \\
\hline 6 & Azygos vein & Energy device & Grasping by forceps & Stapling & Not performed \\
\hline 7 & PA branches & Scissors & Proximal clamp after conversion & Stapling & $\begin{array}{l}\text { To } \\
\text { thoracotomy }\end{array}$ \\
\hline 8 & PA branches & Energy device & $\begin{array}{l}\text { Compression with adjacent lung } \\
\text { after conversion }\end{array}$ & Stapling & $\begin{array}{l}\text { To } \\
\text { thoracotomy }\end{array}$ \\
\hline
\end{tabular}

PA: Pulmonary artery; PV: pulmonary vein. 


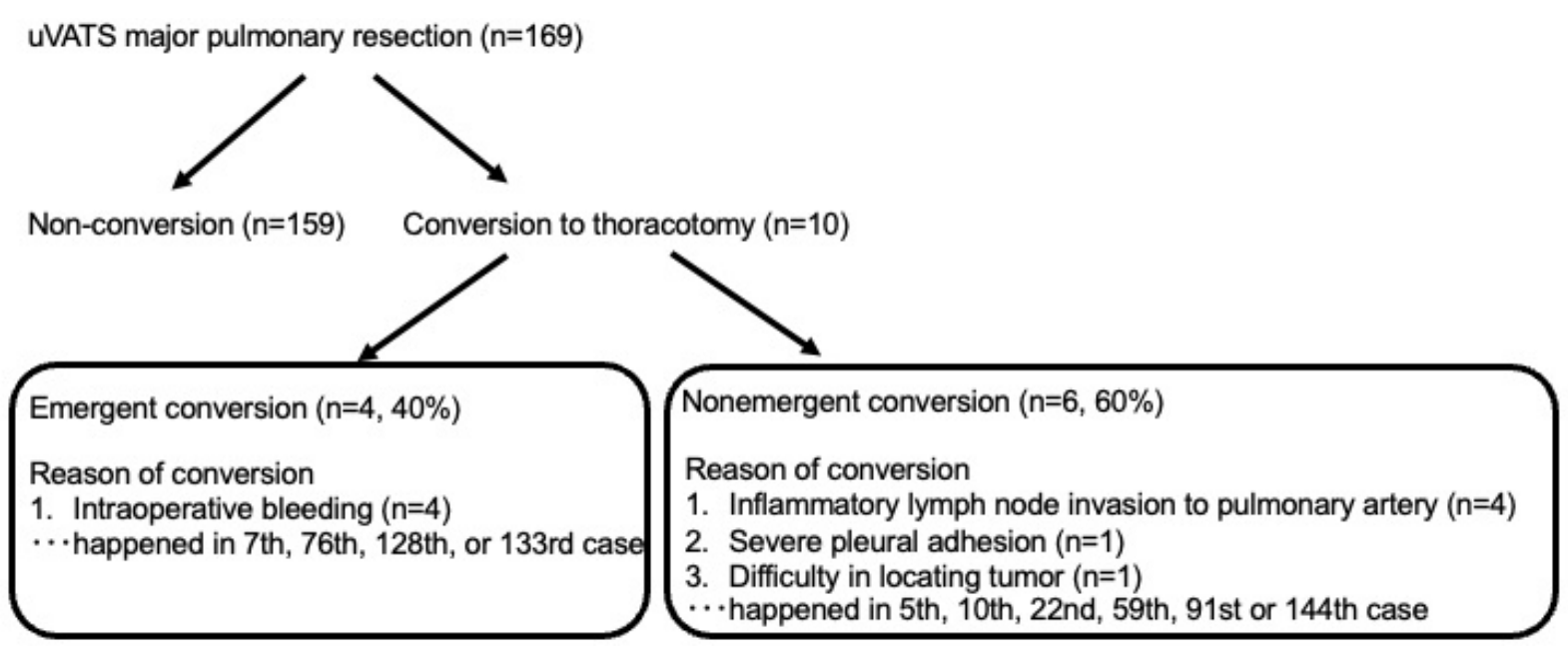

Figure 3. Details of the cases requiring conversion to thoracotomy $(n=10)$.

\section{DISCUSSION}

This study aimed to investigate the efficacy of an algorithm to manage bleeding during uniportal major pulmonary resection. The algorithm needed to be slightly different from the one used by our team for the multiportal approach because of the difficulty in applying thrombostatic sealant directly to the bleeding point to achieve hemostasis. To the best of our knowledge, there have been only 2 studies describing an algorithm to manage bleeding during thoracoscopic uniportal major pulmonary resection ${ }^{[14,15]}$. Scarci et al. ${ }^{[14]}$ initially classified intraoperative bleeding into minor and major types. Subsequently, bleeding management was described for both types, in addition to the timing of conversion to thoracotomy. In Tentzeris's report, the bleeding was classified into three types including pulmonary artery, pulmonary vein, or other uncommon bleeding, and the author finally raised appropriate hemostatic procedures for each type of the bleeding ${ }^{[15]}$. In this study, we focused only on the management of major bleeding. Moreover, we considered this algorithm was an easy-to-apply algorithm, even for surgeons with limited experience, which was important because bleeding complications frequently occur during the initial phase of the surgical learning curve for uniportal thoracoscopy. For example, Xiao et al. ${ }^{[16]}$ used a suction-compression angiography technique to overcome massive bleeding during thoracoscopic surgery. However, we believe that failure to suture the injured point can result in a tear, leading to catastrophic bleeding particularly in cases of injury to the pulmonary artery. Therefore, this technique might not be suitable for surgeons with limited experience.

In this study, no significant group differences were observed for most of the perioperative parameters, except the duration of postoperative drainage and total blood loss. The total blood loss volume in group A was $197 \pm 173 \mathrm{~g}$, which was nevertheless acceptable. Moreover, none of our patients received an intraoperative transfusion and no cases showed catastrophic bleeding, indicating that the management of significant vessel injury during uniportal thoracoscopic major pulmonary resection was appropriate.

In our study, most of the significant vessel injury during uniportal thoracoscopic major pulmonary resection was caused by injury to pulmonary arterial branches, similar to the previous reports on the multiportal approach including ours ${ }^{[17-19]}$. Additionally, the injured vessel was the pulmonary artery branch in all four cases requiring conversion to thoracotomy, indicating that injury to the pulmonary artery in our study may have been life-threatening without appropriate management. The wall of the pulmonary artery is thin and fragile compared to other intrathoracic vessels. Therefore, the pulmonary artery should be treated carefully during the uniportal approach, as with the multiportal approach and thoracotomy. The two cases 
of conversion were caused by mis-stapling of the pulmonary artery. The pulmonary artery was injured by the tip of the stapler when the bronchus in front of pulmonary artery was divided with the stapler [Supplementary Video 1]. The angle of the stapler was relatively limited in the uniportal approach because only a small incision was made ${ }^{[20]}$. A fissureless or unidirectional anterior approach has frequently been adopted for the uniportal approach, with a single incision made at the anterior axillary line, due to the difficulty in obtaining a good surgical view in the posterior area. In the fissureless technique, injury to the pulmonary artery behind the target bronchus caused by mis-insertion of a stapler can lead to massive bleeding, as in our case shown in the video. When a stapling is performed for a pulmonary artery, sufficient exposure to allow for mobilization is important to achieve a safe stapling. For division of pulmonary vein or bronchus, we consider "suction guided stapling" to be very useful, as we have reported in previous studies $^{[21-23]}$.

In this study, emergent conversion was performed in four cases due to injury to the pulmonary artery branch (2.4\% of all cases). The best strategy to manage significant vessel injury is to prevent it from occurring. Moreover, it is important to reduce the rate of emergent conversion. In previous reports, the rate of emergent conversion was $2.4 \%-4.5 \%$ for multiportal thoracoscopic major pulmonary resection, indicating that the uniportal approach does not necessarily increase the rate of emergent conversion compared to the multiportal approach, although the uniportal approach is more technically difficult ${ }^{[11-13]}$.

Previous studies, including those by our team, have demonstrated that the rate of significant vessel injury does not decrease even after gaining thoracoscopic surgical experience ${ }^{[9,10]}$. Emergent conversion occurred in two of the four cases after our team had conducted more than 100 major pulmonary resections via the uniportal approach. These results indicate that injury to the intrathoracic vessels, particularly the pulmonary artery, must be avoided, even after gaining surgical experience in the use of the uniportal or multiportal approach.

This study has several limitations. First, this was a retrospective study including a small number of patients from a single institution. Moreover, the uniportal approach was limited to cT1No primary lung cancer, pulmonary metastasis, or benign disease during the first 30 cases to ensure the safety of the operation, which might bias the results. However, all operations were performed by two highly experienced surgeons, which reduced bias.

In conclusion, we managed significant vessel injury appropriately during uniportal thoracoscopic major pulmonary resection using the troubleshooting algorithm. The algorithm for the uniportal approach was considered effective and easy to apply even by less-experienced surgeons.

\section{DECLARATIONS}

\section{Acknowledgments}

The authors thank all of the surgeons and their coworkers, and the editors and reviewers for their assistance with the manuscript.

\section{Authors' contributions}

Conceptualization, formal analysis, investigation and writing of the original draft: Igai $\mathrm{H}$

Project administration: Kamiyoshihara $\mathrm{M}$

Writing, reviewing, and editing: Matsuura N, Ohsawa F, Numajiri K 


\section{Availability of data and materials}

The data underlying this article will be shared on reasonable request to the corresponding author.

\section{Financial support and sponsorship}

None.

\section{Conflict of interest}

All authors declared that there are no conflicts of interest.

\section{Ethical approval and consent to participate}

This study was approved by the Institutional Ethics Board of Japanese Red Cross Maebashi Hospital (Approval No.: 2021-27, Date: July 26, 2021) and written informed consent was waived.

\section{Consent for publication}

Written informed consent was waived in this study.

\section{Copyright}

(c) The Author(s) 2022.

\section{REFERENCES}

1. Yamamoto H, Okada M, Takada M, Mastuoka H, Sakata K, Kawamura M. Video-assisted thoracic surgery through a single skin incision. Arch Surg 1998;133:145-7. DOI PubMed

2. Migliore M, Giuliano R, Deodato G. Video assisted thoracic surgery through a single port. Proceedings of Thoracic Surgery and Interdisciplinary Symposium on the Threshold of the Third Millennium, Naples, Italy. 2000. p. 29-30.

3. Gonzalez-Rivas D, Delgado M, Fieira E, Mendez L. Single-port video-assisted thoracoscopic lobectomy with pulmonary artery reconstruction. Interact Cardiovasc Thorac Surg 2013;17:889-91. DOI PubMed PMC

4. Bourdages-Pageau E, Vieira A, Lacasse Y, Figueroa PU. Outcomes of uniportal vs multiportal video-assisted thoracoscopic lobectomy. Semin Thorac Cardiovasc Surg 2020;32:145-51. DOI PubMed

5. Zhu Y, Liang M, Wu W, et al. Preliminary results of single-port versus triple-port complete thoracoscopic lobectomy for non-small cell lung cancer. Ann Transl Med 2015;3:92. DOI PubMed PMC

6. Hirai K, Takeuchi S, Usuda J. Single-incision thoracoscopic surgery and conventional video-assisted thoracoscopic surgery: a retrospective comparative study of perioperative clinical outcomes $\dagger$. Eur J Cardiothorac Surg 2016;49 Suppl 1:i37-41. DOI PubMed

7. Al-Ameri M, Sachs E, Sartipy U, Jackson V. Uniportal versus multiportal video-assisted thoracic surgery for lung cancer. J Thorac Dis 2019;11:5152-61. DOI PubMed PMC

8. Magouliotis DE, Fergadi MP, Spiliopoulos K, Athanassiadi K. Uniportal versus multiportal video-assisted thoracoscopic lobectomy for lung cancer: an updated meta-analysis. Lung 2021;199:43-53. DOI PubMed PMC

9. Igai H, Kamiyoshihara M, Yoshikawa R, Ohsawa F, Yazawa T, Matsuura N. Algorithm-based troubleshooting to manage bleeding during thoracoscopic anatomic pulmonary resection. J Thorac Dis 2019;11:4544-50. DOI PubMed PMC

10. Yamashita S, Tokuishi K, Moroga T, et al. Totally thoracoscopic surgery and troubleshooting for bleeding in non-small cell lung cancer. Ann Thorac Surg 2013;95:994-9. DOI PubMed

11. Puri V, Patel A, Majumder K, et al. Intraoperative conversion from video-assisted thoracoscopic surgery lobectomy to open thoracotomy: a study of causes and implications. J Thorac Cardiovasc Surg 2015;149:55-61, 62.e1. DOI PubMed PMC

12. Amore D, Di Natale D, Scaramuzzi R, Curcio C. Reasons for conversion during VATS lobectomy: what happens with increased experience. $J$ Vis Surg 2018;4:53. DOI PubMed PMC

13. Fourdrain A, De Dominicis F, Iquille J, et al. Intraoperative conversion during video-assisted thoracoscopy does not constitute a treatment failure†. Eur J Cardiothorac Surg 2019;55:660-5. DOI PubMed

14. Scarci M, Gonzalez-Rivas D, Schmidt J, Bedetti B. Management of intraoperative difficulties during uniportal video-assisted thoracoscopic surgery. Thorac Surg Clin 2017;27:339-46. DOI PubMed

15. Tentzeris V, Papagiannopoulos K. Uniportal video-assisted thoracic surgery treatment of intra-operative complications. J Vis Surg 2018;4:74. DOI PubMed PMC

16. Xiao ZL, Mei JD, Pu Q, Liu LX. Technical strategy for dealing with bleeding during thoracoscopic lung surgery. Ann Cardiothorac Surg 2014;3:213-5. DOI PubMed PMC

17. Flores RM, Ihekweazu U, Dycoco J, et al. Video-assisted thoracoscopic surgery (VATS) lobectomy: catastrophic intraoperative complications. J Thorac Cardiovasc Surg 2011;142:1412-7. DOI PubMed

18. Fournel L, Zaimi R, Grigoroiu M, Stern JB, Gossot D. Totally thoracoscopic major pulmonary resections: an analysis of perioperative complications. Ann Thorac Surg 2014;97:419-24. DOI PubMed

19. Igai H, Kamiyoshihara M, Ibe T, Kawatani N, Osawa F, Yoshikawa R. Troubleshooting for bleeding in thoracoscopic anatomic 
pulmonary resection. Asian Cardiovasc Thorac Ann 2017;25:35-40. DOI PubMed

20. Gonzalez-Rivas D, Sihoe ADL. Important technical details during uniportal video-assisted thoracoscopic major resections. Thorac Surg Clin 2017;27:357-72. DOI PubMed

21. Igai H, Matsuura N, Kamiyoshihara M. Uniportal thoracoscopic upper division segmentectomy of left upper lobe using a unidirectional anterior approach. Multimed Man Cardiothorac Surg 2020:2020. DOI PubMed

22. Igai H, Kamiyoshihara M. Uniportal thoracoscopic fissureless right lower lobectomy using the suction device trick. Multimed Man Cardiothorac Surg 2019:2019. DOI PubMed

23. Igai H, Furusawa S, Ohsawa F, Yazawa T, Matsuura N, Kamiyoshihara M. Application of "suction-guided stapling" during uniportal thoracoscopic major lung resection. Gen Thorac Cardiovasc Surg 2021. DOI PubMed 